\title{
Retro Hits
}

\author{
The old catalysis literature still has much to offer to the research community. This issue presents a selection of retro \\ News \& Views articles that highlight some key historical developments in the subareas of catalysis.
}

P lanning the next research project is one of the moments of creativity that scientists probably enjoy the most. An idea, then the project takes form, and finally a series of crucial experiments materialises. Enthusiasm and ambition however need to take into account the existing literature and eventually a very simple question emerges: has this been already done? Researchers know well how to navigate the literature to find an answer, and focus their attention on those related studies that may compromise the impact of the nascent project ${ }^{1}$. On the other hand, the very same literature search may provide a confirmation - the lack of similar experiments - and the motivation to put the new idea into practice.

While digging into the literature, we occasionally encounter very old articles, which represented a turning point for a discipline. The relevance of such literature for a new article is certainly variable; different bibliometric studies have tried to estimate how frequently contemporary publications refer to older articles, coming to different conclusions ${ }^{2}$. Independently of trends in the number of recent citations, classic literature gives an invaluable contribution to modern science, not only from a pedagogical point of view: in fact, it provides the scale to appreciate the progress and level of advance of a given field.

\section{"Classic literature gives an} invaluable contribution to modern science, not only from a pedagogical point of view: in fact, it provides the scale to appreciate the progress and level of advance of a

\section{given field"}

In this spirit, this issue of Nature Catalysis features a small collection of retro News \& Views articles - a format that has been used at times by other Nature journals ${ }^{3}$ - that retrospectively analyse important advances in the history of catalysis research. Following the style of our traditional News \& Views articles, they spotlight publications that have played a major role in the development of a discipline, by filling important gaps in the knowledge of a scientific community at a given time, or opening up unexpected possibilities in a field.

The small selection presented in these pages certainly does not do justice to the abundance of literature that makes up the foundation of today's catalysis research. The large variety and diversity of articles that have contributed to the basic principles of mechanistic analysis over the years - no matter whether for catalytic nanoparticles or enzymes - or defined the kinetics of industrially relevant processes, makes any choice somewhat arbitrary. We placed our attention on some of the older literature we have encountered more frequently when assessing incoming submissions and completed the series with topics of interest to the editorial office. On balance, the chosen manuscripts qualitatively map the areas that have been most represented at Nature Catalysis since its launch.

Raffaella Buonsanti talks us through the work of Hori and colleagues who in 1986 identified the ability of copper to catalyse the electrochemical reduction of carbon dioxide into ethylene ${ }^{4}$. The field of electrocatalysis has been flourishing in recent years and the attention on methods for the valorization of $\mathrm{CO}_{2}$ has grown considerably stimulated by the need of practical solutions for the recycling of this prevalent greenhouse gas. In this context, copper remains the metal of choice due to its unique ability to catalyse the formation of $\mathrm{C}-\mathrm{C}$ bonds, thereby allowing access to value-added $\mathrm{CO}_{2}$-based products.

Recovering atmospheric carbon in an attempt to mimic photosynthesis has been keeping scientists busy for many years, but certainly not more than the related idea of converting water into hydrogen and oxygen with the help of light. Elizabeth Gibson revisits the 1972 discovery by Fujishima and Honda ${ }^{5}$, where they reported the ability of titanium dioxide to function as a photoanode for the splitting of water. This contribution represents a milestone in the area of solar fuels and after fifty years still receives attention on social media ${ }^{6}$.

Ammonia synthesis via the Haber-Bosch process is a very mature industrial technology, which plays a central role in the production of nitrogen fertilizers. While the process has been deployed since the beginning of the twentieth century, its full mechanistic picture remained elusive until the 70s. Ping Chen and Jianping Guo explore the journey that brought Gerhard
Ertl and colleagues to unravel the details of ammonia synthesis on iron catalysts ${ }^{7}$. Those findings represent a textbook example of mechanistic studies in heterogeneous catalysis, and still inspire the latest research efforts in the area, which are aimed at the development of superior catalysts for ammonia activation under milder conditions.

On the side of homogeneous catalysis, cross-coupling reactions have effectively established themselves as a first-choice method for the construction of complex organic molecules from simple precursors. The history of such transformations is rich and is characterized by the development of ever more efficient methods for the constructions of C-C bonds. Here, Valentine P. Ananikov recalls the early work by Heck and co-workers that led to the establishment of organopalladium complexes as effective reagents for the coupling of organic halides with olefins ${ }^{8}$.

Last but not least, Maria A. Blasco and Paula Martinez describe the discovery of the activity of telomerase by Elizabeth $\mathrm{H}$. Blackburn and Carol W. Greider'. This extraordinary enzyme, which is responsible for preserving the structure of the telomere - the ends of the chromosome - has fascinated biochemists for decades and has far-reaching implications for the treatment of genetic diseases.

Certainly, some of our readers will be already familiar with the literature discussed in this collection of retro News \& Views articles. Nevertheless, we believe that these and other classic studies have not exhausted their initial inspirational power. We hope therefore that by revisiting them with modern eyes they may once more enrich the science they have contributed to develop.

Published online: 21 September 2021 https://doi.org/10.1038/s41929-021-00681-3

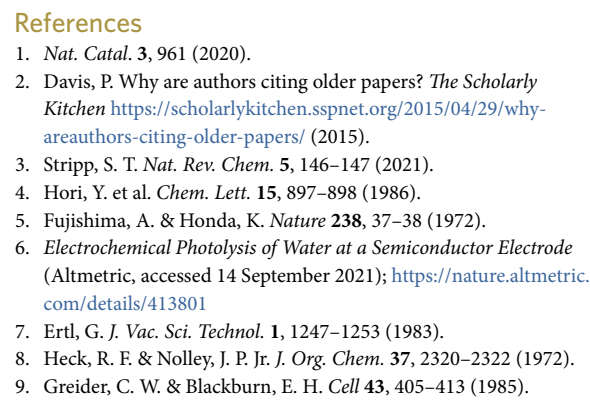

